\title{
Energy Storage Applications for Distribution Grid Support: Composition of Controlling Strategies and a Standardized Model
}

\author{
Istvan Taczi \\ MSc. Student \\ Department of Electric Power Engineering \\ Budapest University of Technology and Economics \\ Egry József Str. 18. - 1111 Budapest (Hungary) \\ Phone number:+36-30/5166473 \\ e-mail: taczi.istvan@eszk.org
}

\author{
Dr. Istvan Vokony \\ Senior Lecturer \\ Department of Electric Power Engineering \\ Budapest University of Technology and Economics \\ Egry József Str. 18. - 1111 Budapest (Hungary) \\ Phone number: +36-30/640-9013 \\ e-mail: vokony.istvan@vet.bme.hu
}

\begin{abstract}
Distribution grids will basically change in the near future: the increasing penetration of renewable energy causes high percentage of intermittent and decentralized generation. This procedure will lead to problems which are unknown for a conventionally planned and operated grid. Bidirectional power flows, power quality issues and voltage sags can occur.

Energy storage systems (ESSs) are able to play a key role in the solutions. With a well-developed controlling strategy, these applications could support the low voltage grids locally: virtual islanding operations and load following, or improved reliability with reserves are both feasible. ESSs can even be used for trading on the liberalized electricity market.

The focus of this work is to build up a modelling method and an analysing script, to test some self-composed controlling algorithms. The simulation considerate the main parameters of an ESS: efficiency, capacity, response time, cycle lifetime in optional time steps through load flow simulations. If a grid model meets a few requirements, it can be used as an input for the simulation script. With further standardizing, this program could be a useful tool for the distribution system operators (DSOs) and network developers.

The results which contain all the relevant electrical power system parameters on an optional timescale could be exported for further evaluation.
\end{abstract}

\section{Key words}

Energy storage, controlling strategies, intermittent generation, economical feasibility, improved reliability, renewable penetration

\section{Introduction}

The energy sector is changing worldwide, because of the emission reduction aims. Renewable energy sources are getting more and more popular. The Compound Annual Growth Rate (CAGR) of PV installations was $44 \%$ between 2000 and 2014. The purpose is clear: the world must lean on clean energy. This distributed generation will need a more flexible and balanced grid with wellprovided backup reserves. Conventional controlling methods are insufficient to provide the quality of supply with the new constraints. New trends will emerge to evolve a sustainable power system for the future.

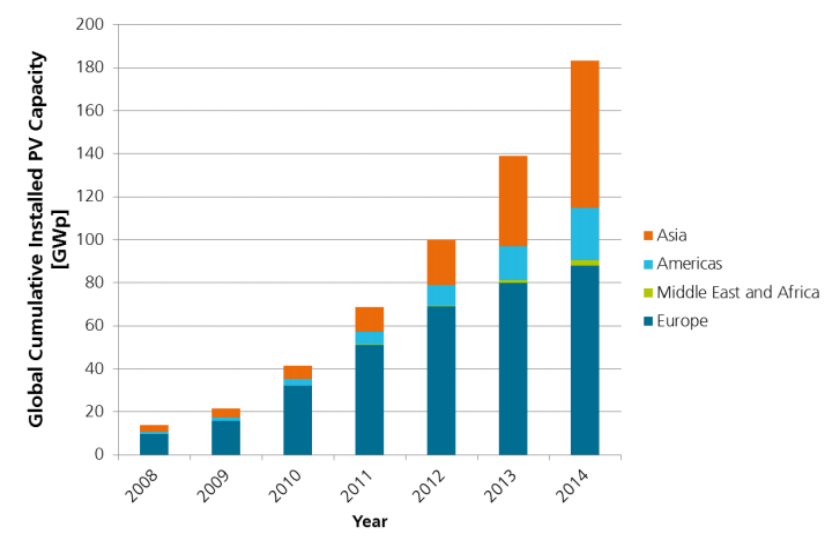

Fig 1.: Global cumulative PV installation until 2014 [1]

Energy storage systems (ESSs) can provide a variety of application solutions along the entire value chain of the electrical system, from wholesale services to transmission and distribution support to end-customer uses. By setting aside it can decouple supply from demand, and also increasing the system flexibility and improving reliability and asset utilization. Just as transmission and distribution systems can move electricity over distances to end users, energy storage systems can move electricity through time, providing it when and where it is needed.

The focus of this work is to create a simulation model which can implement controlling strategies with ESSs and quantify the effects of an application. The modelling methods are mostly standardised, so the simulation script can generate calculation data from an arbitrary grid topology. There are input data for each technology and 
application to help the user in the first steps. Based on the collected data, the strategies published here are tested in real life environment in Fót city, Hungary. After the validation process throughout the measurements, an evaluation proceeding should be added to make the output data more utilizable. The whole project could help decision makers and network developers to make reasonable investments.

\section{Modelling method}

\section{A. The demonstrational input grid parameters and the software environment}

The simulation software is implemented in DigSilent Power Factory v14. There are a lot of basic scripts and objects which are useful for a correct and perspicuous input framework. The program is running as a load flow script and using the built-in Newton-Rapshon algorithm. The user can set all the main variables in connection with an energy storage equipment as it can be seen below.

Table I. - Input parameters of the simulation

\begin{tabular}{|l|c|c|}
\hline \multicolumn{1}{|c|}{ Name } & Dim. & Function \\
\hline Start & hour & Starting Time \\
\hline Step & hour & Time resolution \\
\hline Stop & hour & Stopping time \\
\hline StorageStrategy & integer & Algorithm choosing \\
\hline Efficiency & $\%$ & Round-trip efficiency \\
\hline Energy & MWh & The starting energy \\
\hline Response & hour & Response time \\
\hline Sizing & T/F & Sizing methodology \\
\hline MidPrice & $\$ / \mathrm{MWh}$ & Median of the price curve \\
\hline UpLimit & $\$ / \mathrm{MWh}$ & Selling limit \\
\hline LowLimit & $\$ / \mathrm{MWh}$ & Buying limit \\
\hline MaxCycle & integer & Lifetime \\
\hline Power & MW & Nominal power \\
\hline Capacity & MWh & Nominal capacity \\
\hline SwitchOff & hour & Switches off an element \\
\hline SwitchBack & hour & Switches on an element \\
\hline
\end{tabular}

The pilot grid has an external grid object, a 20/0.4 kV transformer (160 kVA), locally installed photovoltaic systems (37 kW, 2 installations), wind generator $(20 \mathrm{~kW}$, currently out of service due to inverter problems), a hydro power generator (small scale, $200 \mathrm{~W}$ ) and 8 consumers modelled as general loads. These loads represent some of the main residential curves, such as sprinklers, heat pump and lighting. There is an office on this territory. The peak local demand is approximately $55 \mathrm{~kW}$. There is also a chance for demand side management strategy implementation: the sprinklers (which has the largest nominal power from the loads) switching is happening in identified time so these large differences will not spoil the balancing.

\section{B. Restrictions for effective modelling}

All of the generators must be static generator type, because the program has to identify every one of them on an arbitrary grid. Each objects are equipped with characteristics from real-life measurements. The grid is equipped with a local SCADA system, which can be used for both measurement and controlling. Stochastic models are also feasible, there are no restrictions for the characteristics. The biggest limit of this script is the number of elements, but it can be set through the code if needed. The script can handle only one ESS, so it must be a concentrated element, with the expression "ESS" in its name for identification. The elements must have at least an active power curve, but power factor or reactive power trends are also suggested. Nominal power data should be added for the sizing algorithm. Resistance, reactance and short-circuit data could be useful for a more realistic model, and even for voltage and power loss calculations. All the main variables of a power grid are accessible through the software's object element set for the evaluation.

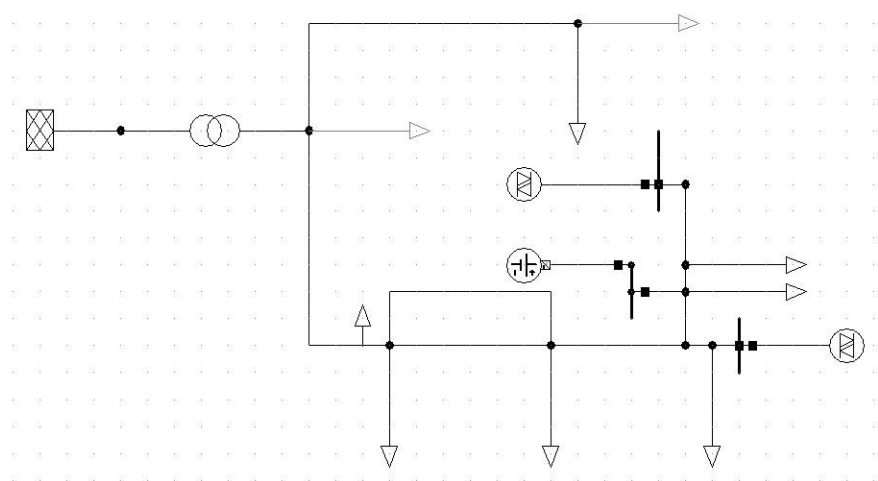

Fig. 2.: Final state of the input grid topology: the external grid and transformer on the left, the loads represented as triangles. The ESS, solar panels and the water generation are static generators.

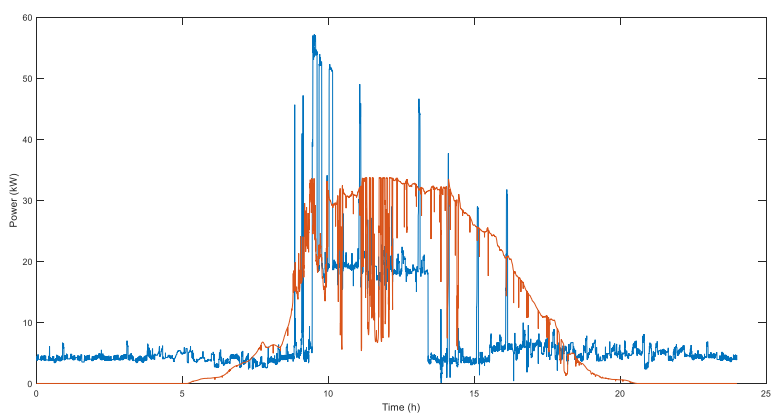

Fig. 3.: Total local consumption (blue) and total local generation (orange): this figure represents the characteristics of the grid

\section{Consideration of ESS parameters}

To limit the restrictions for the input network model, the ESS is a static generator. A special energy status estimation is implemented which could be used for defining both capacity constraints and sizing methods. It calculates the energy for the next load flow iteration step from the actual energy and the adjusted power. If it violates the limits (energy below zero or higher than the nominal capacity) the actual power settings are being overwritten before the time step. 
This might mean that safety limits should be considered in the capacity constraint input.

Another main parameter is the cycle lifetime of an ESS. This variable is monitored through two variables: both the switches in the output power and the full charges and discharges are counted. Change of efficiency could be implemented through this variable, and with an upper limit of the summarization, the lifetime could be considered.

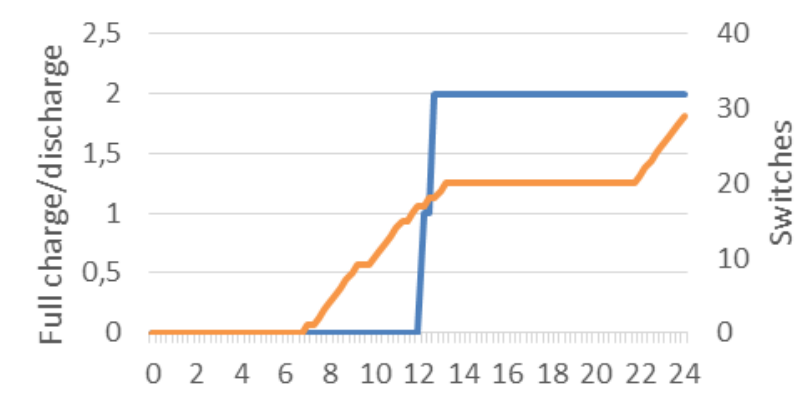

Fig. 4.: Counting of switches and constraint reaches: these variables could be used for representing the lifetime and aging of the installation

Efficiency is an important parameter for the applications. This modelling method takes it into consideration with a concentrated calculation: through the charging, the energy of the ESS increases with the product of the efficiency, power and the time (the set power in a time step is constant). The efficiency is an input parameter, but could be changed if aging of elements is also considered.

Response time of the ESS is also a crucial thing and the script can handle it: if the time step is longer than the response time then the controlling algorithm is slower than the technology. When the response time is longer, the change determined in the actual point takes place in actual time+response time. Meanwhile the calculation of the load flow is still going, but the interaction is delayed.

\section{Simulation results}

First all the relevant information gathered by the script and it is saved to objects (the DigSilent Programming Language is object-oriented) with a filtering selection. After that it identifies the ESS from its name (it is also a static generator type like all the suppliers) to get the subject of the controlling algorithms. The built in load flow script runs with the given time premises, and actualize the variables of the objects in every step.

When all of the data is refreshed, the controlling strategies take over. Following the calculations, the results are being saved to a result file and getting printed to the output as well.

The capabilities of this method are being shown through the demonstrational grid. All of the data is provided by a distribution system operator from real-life measurements.

\section{A. Virtual islanding operation}

The aim of the first strategy is realize islanding operation within the local microgrid. This scenario is important for critical infrastructures, isolated grids and can be used when an outage occur. All strategies shown below start with a fully charged ESS but it can be changed to any percentage on the graphic user interface. The simulation period covers a full day from midnight to midnight. The time scale and the duration could be set through the input parameters so it is feasible to run longer simulations with appropriate data.

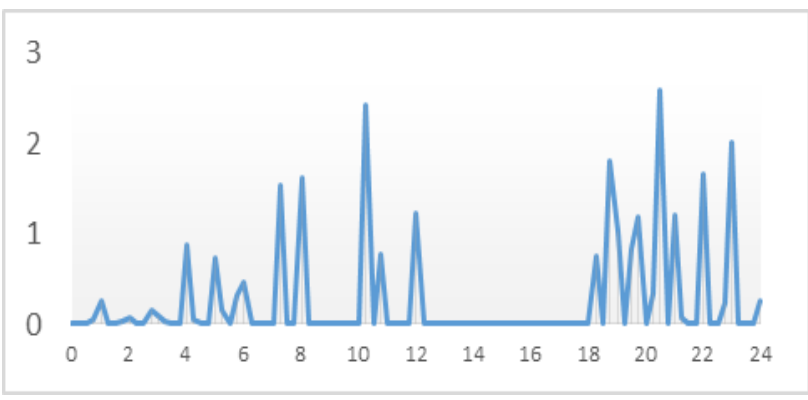

Fig 5.: Power consumption from the external grid in virtual islanding operation $\mathrm{kW}(\mathrm{h})$

Figure 5 shows that this microgrid could work without significant power consumption from the external grid. With these characteristics even the maximum demand is below $3 \mathrm{~kW}$.

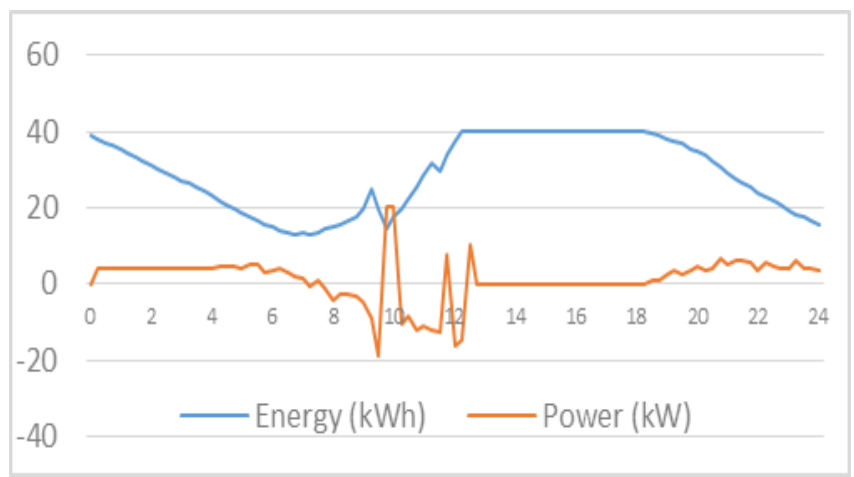

Fig 6.: ESS energy and power curve in virtual islanding operation

The ESS characteristics on figure 6 show that there is a local over-generation, and the ESS has insufficient capacity to store all the generated solar energy. The local grid is heavily relying on that, so the critical time interval might be the night on cloudy days.

\section{B. Energy trading on the liberalized electricity market}

The ESS can work to fulfil economical purposes. In this case, two energy trading strategies will be shown. 


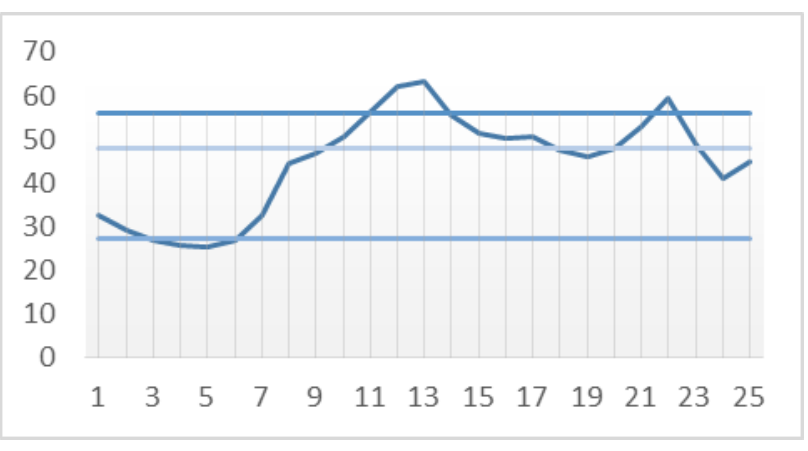

Fig 7.: Energy price in $€ / M W h$ from the Hungarian Power Exchange (HUPX) company

The input (figure 7) of those strategies is a price curve and the decision thresholds. This data is gathered from the Hungarian Power Exchange company, an average price from July, 2013, when the solar penetration is exceptionally high.

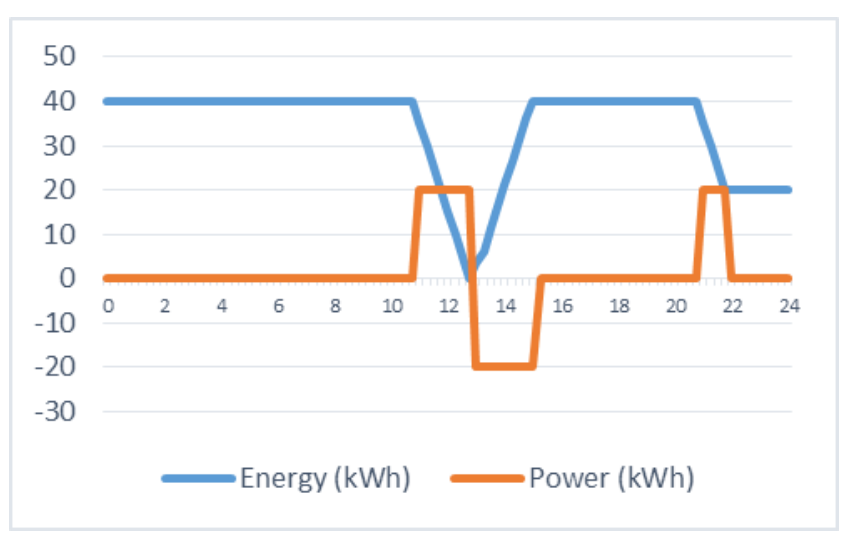

Fig 8.: Energy trading with buy and sell limits

The first strategy (figure 8 ) only sell when the price is above $56 € / \mathrm{MWh}$. When the storage got empty and the price went below the selling limit, there was an overgeneration (charging state from $13 \mathrm{~h}$ to $15 \mathrm{~h}$ ). The buying price is $27 € / \mathrm{MWh}$, so the ESS start to charge from the external grid when the price gets below that threshold.

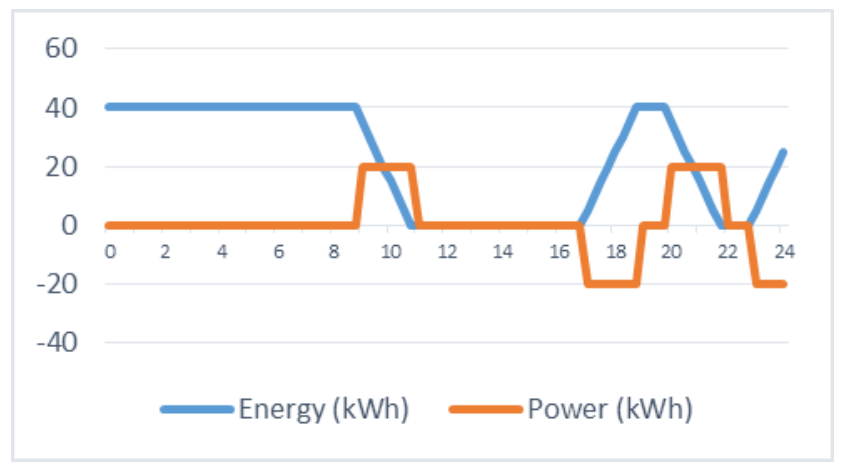

Fig 9.: Energy trading with mean price

The second strategy (figure 9) has a mean value threshold: the ESS is charging when the price is below that threshold and the stored energy did not reach the capacity yet, and discharges when the price is above and the energy is not zero. The capacity constraint is expressive on this diagram.
These strategies should be evaluated from an economical viewpoint. Beside that the effects on the power grid have to be taken into the count.

\section{Reliability reserve operation}

The ESS plays a backup reserve role for the main supplier of the grid in the third strategy (fault ride through capability). The program is able to switch generators on and off, so outages can be modelled this way. The implemented strategy is checking the status of the main supplier (solar panels on this grid): if it is disconnected from the grid, the ESS starts a load following discharge.

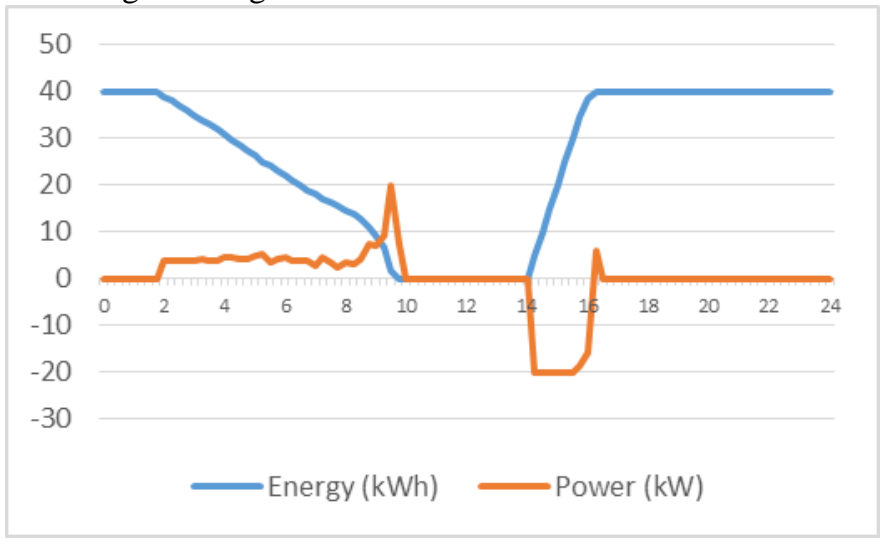

Fig 10.: ESS serving as a reserve: outage (2h) and start (14h) of the supplier

As it can be seen on figure 10, there was an outage at 2:00 AM, and then the ESS is in a discharge state. If there is overproduction, it still can charge, because the algorithm calculates the actual net load flow in every step, and set the output to compensate it. The time of operation is maximized with this load following concept. The script is able to monitor more generators simultaneously. When the generator got connected to grid again the charging from the local over-generation began. It is an alternative that the ESS can be charged from the external grid to be at full capacity faster.

\section{Reactive power compensation}

Reactive power is a crucial variable on power grids: if there is too much of it, the losses are increasing significantly. Lot of companies working on more efficient VAR compensation and power quality issues and there are countless solutions. The main advantage of ESS is that it can provide the reactive power compensation in an aggregated operation to cumulate the benefits of applications. 


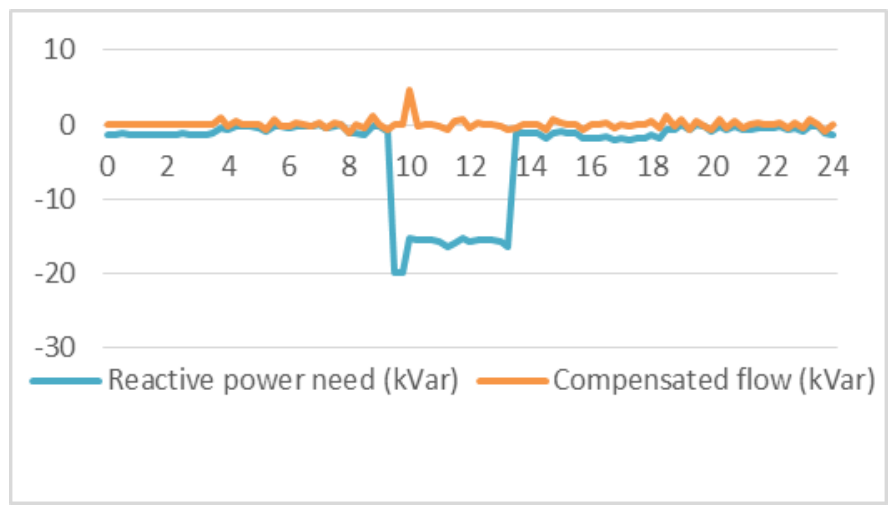

Fig. 11.: Reactive power compensation

Figure 11 represents the output of the strategy is a compensated flow with a peak of $5 \mathrm{kVar}$, which is far less than the starting state. The high amount of reactive power consumed by the sprinkler motors is almost fully compensated in the daytime.

\section{E. Sizing algorithms}

A useful capacity sizing function is also implemented. The energy constraint specified above had to be restructured: the variable is the maximum needed energy, the starting state is 0 . Then it follows the power adjustments (practically it is the integral of that function with a -1 multiplier). The return value is the extrema of that curve, this is the needed capacity. This should be oversized with an input factor from the user.

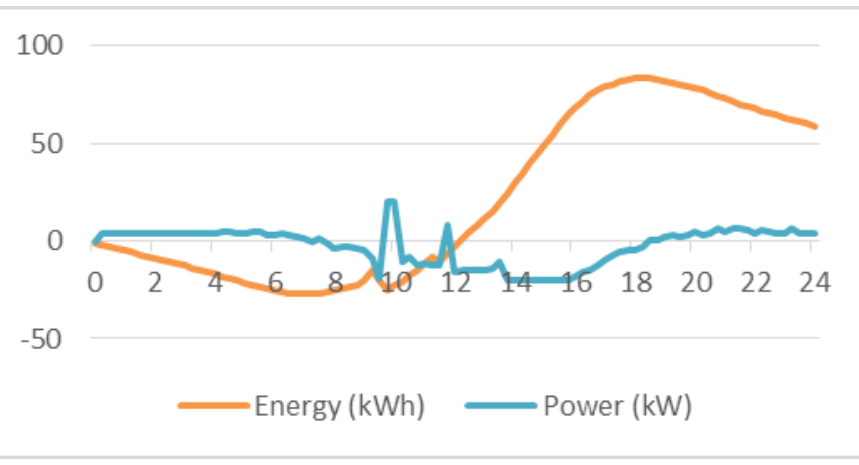

Fig. 12.: Sizing algorithm in virtual islanding operation

It can be seen on figure 12 that through this sizing algorithm for virtual islanding operation that the storage needed for the photovoltaic generation in the afternoon is the determining constraint. This sizing method is implemented for all the strategies shown to estimate the needed capacity. With a stochastic (or longer time span) data it is a very useful tool for network development planning even if no ESS will be set.

\section{Evaluation of the generated data}

The focus was a composition of a modelling method and useful strategies to make the effects quantifiable. This evaluation method is a simplified process which just focuses on the bulk effects of ESSs.

\section{A. Technical parameters}

The objective functions are optimizing the grid in some way and there are technical benefits from that. Improved reliability and stability is achievable. The grid can work in standalone operation or the losses can be reduced.

Table II. - Technical parameter changes with controlling

\begin{tabular}{|c|c|c|}
\hline Controlling state & Energy (kWh) & Power (kW) \\
\hline Virtual islanding without control & 63,61 & 20 \\
\hline $\begin{array}{c}\text { Virtual islanding with the } \\
\text { controlling }\end{array}$ & 16,96 & 2,7 \\
\hline $\begin{array}{c}\text { Controlling state } \\
\text { Reactive power flow of the grid }\end{array}$ & $-78,36$ (ind.) & 20 (ind.) \\
\hline $\begin{array}{c}\text { Compensated reactive power flow } \\
\text { Energy (kVar) }\end{array}$ & Power (kVar) \\
\hline
\end{tabular}

The summarization is verifying the fundamental ideas behind the controlling strategies. In this demonstrational decentralized distribution grid, the local generation provides flexibility. The reactive power flow could be held under strict limits. The technical background of the strategies is well-represented in the figures shown above, these could be used for further evaluation.

\section{B. Economic feasibility: return on investment calculations}

The spreading of ESSs depends on the economic effects. The targets of regulators could be helpful in the near future for ESS applications for renewable energy support. Also the deceleration of prices (automation in industry, maturing technologies, bulk producing) will facilitate ESS applications.

This simplified return on investment calculation shows a possible way of the evaluation: the positive changes are concentrated in one parameter, the electricity price (the ratio of the price/cost is important, so the ratio changes could be unified in the price). In this case $10 \%$ of price changes is estimated. The cost data is provided by a recent study of the levelized cost of energy storage (marked as a reference) which has an estimation for the future: the rough $10 \%$ deceleration is acceptable from those data and other projections from industrial stakeholders. The costs are divided to capital and operation / maintenance costs. The benefit is the income realized from the sold energy on the liberalized market. The one day timespan data (and also the one day timespan prices) are extrapolated for a 15 year span. 
Table III: Parameters for return on investment calculations

\section{Parameters}

\begin{tabular}{|lr|}
\hline Power [kW] & 20 \\
Capacity [kWh] & 100 \\
Capital /kWh & 550 \\
O\&M /kWh & 12 \\
\hline & 95000 \\
\hline Total Cost of investment (USD) & 55000 \\
\hline \hline Capital & 40000 \\
\hline Solar system & 1200 \\
\hline & \\
\hline \hline & \\
Income & \\
From trading on the liberalized electricity market & 11,61 \\
(USD/Day)
\end{tabular}

The solar system is also included: this step is made for to ease the calculations, because in this scenario the energy which could not be stored due to the capacity constraint is also a benefit (by selling it on the liberalized electricity market). This model is a rough approximation, but from these data it is a reasonable method. Longer time spans would be far utilizable for economic effect examination. The parameters are for a Lithium-ion battery for microgrid and virtual islanding operations and distribution services.

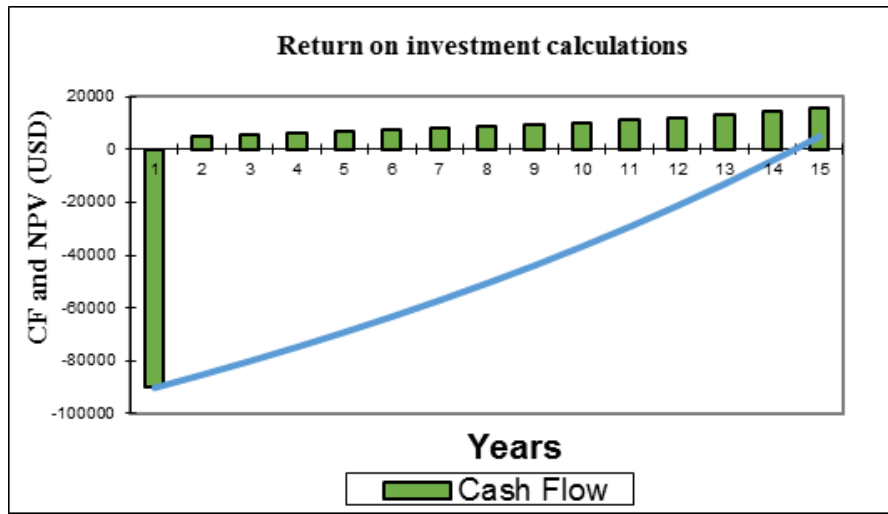

Fig 13.: Return on investment calculations for the energy trading strategy with mean price

As Figure 13. shows, the return on investment could be feasible with these constraints. The battery lifetime is critical, but the low-cost approximation includes the replacement costs on both the AC and DC sides. From those simulations, the conclusion is that energy storage system applications can be competitive in both technical and economic viewpoint with proper local circumstances and effective controlling strategies.

\section{Conclusion}

The program is capable of implementing a standard and detailed analysis on the optimization of an ESS. The effects could be quantified by the generated load flow data. Even more algorithms could be written such as voltage stability support or transmission and distribution loss reduction. With more adaptive and predictive elements the operation will converge to the ideal. Further development opportunities could make the modelling more complex: it should take the physical size in to account because this parameter may be critical for some applications. Choosing the optimal placement from some possible positions would also be a useful feature.

The whole software could be validated through measurements soon. The provider of the measurement data (ELMÜ, Hungarian distribution system operator) is also working on the virtual islanding of this grid, so the measurement data and the algorithm could be used in a real life environment. Three strategies was tried on the grid to gather measurement data. The energy trading with high and low limits was also tried, such as the reliability function. The algorithms implemented in the SCADA controlling system are not different from the shown which are shown above but written in the Citect CiCode Scada language so there is a direct industrial connection of this work. The controlling worked well. Further measurements and optimizing is planned.

This methodology should be used by the DSOs and any other stakeholders who are planning a network development as a tool to consider the opportunities of ESSs. Through the evaluation process the economic feasibility could be examined.

\section{References}

[1] Fraunhofer Institute: Photovoltaics Report, 2015.

[2] International Renewable Energy Agency: Electricity storage and renewables for island power, 2012.

[3] Electric Power Research Institute: Electricity energy storage technology options, 2010.

[4] International Energy Agency: The future role and challenges of energy storage working paper, 2014.

[5] International Electrotechnical Commission: White paper on energy storage, 2011.

[6] International Journal of Electrochemical Science: An intelligent control strategy of battery energy storage system for microgrid energy management under forecast uncertainties [7] stoRE, Final publishable report of the European Union, 2014.

[8] European Technology Platform: Strategic Deployment Document for Europe's Electricity Networks of the future, 2013.

[9] DigSilent Power Factory: DigSilent Programming Language User Manual, DigSilent GmbH, 2003.

[10] Citect SCADA: CiCode Reference, Schneider Electric

[11] International Energy Agency: Technology roadmap on energy storage, 2014.

[12] Lazard: Levelized cost of energy storage analysis, 2015.

[13] Eurelectric: Decentralized storage: impact on future's distribution grids

[14] U.S. Department of Energy and Electric Power Research Institute: Electricity storage Handbook in collaboration with NRECA 